\title{
Removing the barrier to the calculation of activation energies
}

Oluwaseun O. Mesele, and Ward H. Thompson

Citation: The Journal of Chemical Physics 145, 134107 (2016);

View online: https://doi.org/10.1063/1.4964284

View Table of Contents: http://aip.scitation.org/toc/jcp/145/13

Published by the American Institute of Physics

\section{Articles you may be interested in}

Removing the barrier to the calculation of activation energies: Diffusion coefficients and reorientation times in liquid water

The Journal of Chemical Physics 147, 134103 (2017); 10.1063/1.4997723

Simulations of the infrared, Raman, and 2D-IR photon echo spectra of water in nanoscale silica pores

The Journal of Chemical Physics 144, 194709 (2016); 10.1063/1.4949766

Communication: Inverse design for self-assembly via on-the-fly optimization

The Journal of Chemical Physics 145, 111101 (2016); 10.1063/1.4962754

Perspective: Kohn-Sham density functional theory descending a staircase

The Journal of Chemical Physics 145, 130901 (2016); 10.1063/1.4963168

Water-anion hydrogen bonding dynamics: Ultrafast IR experiments and simulations

The Journal of Chemical Physics 146, 234501 (2017); 10.1063/1.4984766

Perspective: Machine learning potentials for atomistic simulations

The Journal of Chemical Physics 145, 170901 (2016); 10.1063/1.4966192

\section{AIP | The Jounal of Chemical Physics}




\title{
Removing the barrier to the calculation of activation energies
}

\author{
Oluwaseun O. Mesele and Ward H. Thompsona) \\ Department of Chemistry, University of Kansas, Lawrence, Kansas 66045, USA
}

(Received 12 June 2016; accepted 21 September 2016; published online 6 October 2016)

\begin{abstract}
Approaches for directly calculating the activation energy for a chemical reaction from a simulation at a single temperature are explored with applications to both classical and quantum systems. The activation energy is obtained from a time correlation function that can be evaluated from the same molecular dynamics trajectories or quantum dynamics used to evaluate the rate constant itself and thus requires essentially no extra computational work. Published by AIP Publishing. [http://dx.doi.org/10.1063/1.4964284]
\end{abstract}

\section{INTRODUCTION}

It is now common to calculate reaction rate constants using time-correlation functions (TCFs) evaluated from molecular dynamics (MD) simulations that capture reactive trajectories. ${ }^{1-8}$ However, further information about the reaction is generally obtained by additional simulations. A key example is the corresponding reaction activation energy that is typically obtained by constructing an Arrhenius plot based on the rate constant, $k(T)$, calculated from simulations at different temperatures. However, this approach belies the fact that the ensemble of reactive trajectories obtained in a MD simulation actually contains considerably more information.

Dellago and Bolhuis ${ }^{9}$ have shown that the activation energy can be calculated directly in the context of transition path sampling calculations. They applied it to a model isomerization reaction and others have employed it to determine the activation energy in other systems using transition path sampling. ${ }^{10-12}$ In this paper, we extend this approach by showing that the activation energy can be obtained directly from the trajectories used to obtain the rate constant at a single temperature using other TCFs. In addition, the same tactic can be applied to calculate the activation energy within quantum mechanical TCF formulations of the reaction rate constant. Thus, these approaches can be implemented in the context of nearly any method for calculating a rate constant. We apply the results to determining the activation energy of the one-dimensional Eckart barrier, described in terms of both classical and quantum mechanics, and the classical hydrogen-bond (H-bond) exchange dynamics in liquid water.

\section{TIME CORRELATION FUNCTIONS AND ACTIVATION ENERGY}

\section{A. Classical}

For the purposes of illustration, we first consider the reactive flux (or flux-side) TCF expression for the rate constant

$$
k=\lim _{t \rightarrow \text { long }} C_{f s}(t)=\lim _{t \rightarrow \text { long }}\left\langle F_{s}(0) \theta\left[s(t)-s^{\dagger}\right]\right\rangle .
$$

\footnotetext{
${ }^{a)}$ Electronic mail: wthompson@ku.edu
}

Here a dividing surface at $s=s^{\ddagger}$ is assumed to separate reactants $\left(s<s^{\ddagger}\right)$ and products $\left(s>s^{\ddagger}\right), F_{s}(0)=\delta\left[s(0)-s^{\ddagger}\right] v_{s}(0)$ is the classical flux through the dividing surface at $t=0$ with $v_{s}$ the velocity in the reaction coordinate, $s$, direction, and $\theta(s)$ is the Heaviside step function. The rate constant is given by the "long"-time limit, which indicates times long enough for trajectories initiated at the dividing surface to have reached the products, but shorter than the time scale for the products formed to convert to reactants via the reverse reaction. ${ }^{5}$

The activation energy is given by the temperature dependence of the rate constant as

$$
E_{a}=-\frac{d \ln k}{d \beta}
$$

which, using Eq. (1), gives

$$
E_{a}=-\frac{1}{k} \lim _{t \rightarrow \text { long }} \frac{d C_{f s}(t)}{d \beta} .
$$

It is important to note that $E_{a}$, which is defined by Eq. (2), is distinctly different from the free energy barrier for the reaction. We can write the TCF in a more explicit form as

$$
C_{f s}(t)=\frac{1}{Q_{r}} \operatorname{Tr}\left[e^{-\beta H} F_{s}(0) \theta\left[s(t)-s^{\dagger}\right]\right],
$$

where $Q_{r}$ is the reactant partition function, $\operatorname{Tr}$ represents an average over all phase space initial conditions, $\beta=1 / k_{b} T$, and $H$ is the Hamiltonian. Then, it is clear from this expression that $C_{f s}(t)$ depends on temperature, and hence $\beta$, only through $Q_{r}$ and the Boltzmann factor, $e^{-\beta H}$. Thus,

$$
\begin{aligned}
\frac{d C_{f s}(t)}{d \beta}= & -\frac{1}{Q_{r}} \operatorname{Tr}\left[e^{-\beta H} H(0) F_{s}(0) \theta\left[s(t)-s^{\ddagger}\right]\right] \\
& -\frac{d \ln Q_{R}}{d \beta} C_{f s}(t) .
\end{aligned}
$$

However, $-d \ln Q_{R} / d \beta=\langle H\rangle_{r}$ is the average reactant energy, which allows the two terms in the above equation to be combined as

$$
\frac{d C_{f s}(t)}{d \beta}=-\left\langle\delta H(0) F_{s}(0) \theta\left[s(t)-s^{\ddagger}\right]\right\rangle,
$$

where $\delta H(0)=H(0)-\langle H\rangle_{r}$ is the fluctuation of the energy from the average reactant value. This gives the activation 
energy as

$$
E_{a}=\frac{1}{k} \lim _{t \rightarrow \text { long }}\left\langle\delta H(0) F_{s}(0) \theta\left[s(t)-s^{\ddagger}\right]\right\rangle .
$$

This is the result derived previously by Dellago and Bolhuis ${ }^{9}$ and it shows that the activation energy can be obtained directly from a TCF that can be evaluated in the same simulation as that used to obtain the rate constant itself. The physical meaning is also clear as what is required is the addition of the energy fluctuation (relative to that of the reactants) at the transition state dividing surface to the reactive flux correlation function, Eq. (1). In this way, the present result echoes the interpretation of the activation energy first proposed by Tolman nearly a century ago. ${ }^{13}$

This approach for obtaining direct time correlation function expressions for the activation energy is general. An analogous result can be obtained for the flux-flux TCF,

$$
C_{f f}(t)=\left\langle F_{s}(0) F_{s}(t)\right\rangle,
$$

the integral of which gives the rate constant

$$
k=\int_{0}^{\infty} C_{f f}(t) d t .
$$

An expression for the activation energy is obtained, using Eq. (2) and the same approach applied above for the flux-side TCF, as

$$
E_{a}=\frac{1}{k} \int_{0}^{\infty}\left\langle\delta H(0) F_{s}(0) F_{s}(t)\right\rangle d t,
$$

in close analogy to Eq. (7).

The same approach can be applied to the rate constant obtained within the stable-states picture ${ }^{6,7}$ in which the rate constant is obtained from the side-side ${ }^{4} \mathrm{TCF}$

$$
\begin{aligned}
C_{r p}(t) & =\left\langle n_{r}(0) n_{p}(t)\right\rangle \\
& =\frac{1}{Q_{r}} \operatorname{Tr}\left[e^{-\beta H} \theta\left[s_{r}-s(0)\right] \theta\left[s(t)-s_{p}\right]\right],
\end{aligned}
$$

where $r$ and $p$ represent reactants and products, respectively. Here, $n_{r}(0)=\theta\left[s_{r}-s(0)\right]=1$ when the system is in the reactant well at time $t=0$ and $n_{r}(0)=0$ otherwise; $n_{p}(t)$ $=\theta\left[s(t)-s_{p}\right]=1$ when the system is in the product well at time $t$ and $n_{p}(t)=0$ otherwise. Thus, $C_{r p}(t)$ is zero at $t=0$ and rises to one with the time scale for converting reactants to products. The rate constant is equal to the time derivative of $C_{r p}(t)$ at times long relative to the time scale for passing between the two dividing surfaces but much shorter than $1 / k .^{4,5}$ In practice, however, the rate constant is often obtained by the more global description, $1-C_{r p}(t)=e^{-k t}$, a form that is rigorously obeyed only for times longer than the transit time between the reactant and product regions. ${ }^{14}$ In many (but certainly not all) cases, however, this single exponential form is found to be a good approximation for all times and we will adopt it in the following for the purposes of illustration. The rate constant can then be obtained as

$$
k^{-1}=\int_{0}^{\infty}\left[1-C_{r p}(t)\right] d t,
$$

such that the activation energy is given by

$$
E_{a}=-k \int_{0}^{\infty} \frac{d C_{r p}(t)}{d \beta} d t
$$

Using Eq. (11) then gives the activation energy as

$$
E_{a}=k \int_{0}^{\infty}\left\langle\delta H(0) n_{r}(0) n_{p}(t)\right\rangle d t,
$$

in close correspondence to the results for the reactive flux TCFs derived above.

\section{B. Quantal}

The quantum mechanical reaction rate constant can be obtained by analogous time-correlation functions, ${ }^{3,4}$ including a flux-side TCF,

$$
\begin{aligned}
k_{q m} & =\lim _{t \rightarrow \text { long }} C_{f s}(t) \\
& =\lim _{t \rightarrow \text { long }} \frac{1}{Q_{r}} \operatorname{Tr}\left[e^{-\beta \hat{H}} \hat{F}_{s} \hat{\mathcal{P}}(t)\right] .
\end{aligned}
$$

Here, $C_{f s}(t)$ is the quantum mechanical flux-side TCF, Tr is now a quantum mechanical trace, $\hat{H}$ is the Hamiltonian operator, $Q_{r}$ is the quantum mechanical reactant partition function, and $\hat{F}_{s}=i\left[\hat{H}, \theta\left(\hat{s}-s^{\ddagger}\right)\right] / \hbar$ is the flux operator through the dividing surface separating reactants and products. The operator $\hat{\mathcal{P}}(t)$ projects onto reactive space and can take various forms. ${ }^{3,4}$ The one most analogous to Eq. (1) is

$$
\hat{\mathcal{P}}(t)=e^{i \hat{H} t / \hbar} \theta\left(\hat{s}-s^{\ddagger}\right) e^{-i \hat{H} t / \hbar} .
$$

It is convenient to use the fact that, in the long-time limit, $[\hat{H}, \hat{\mathcal{P}}(t)]=0,{ }^{4}$ so that, at long times,

$$
C_{f s}(t)=\frac{1}{Q_{r}} \operatorname{Tr}\left[e^{-\beta \hat{H} / 2} \hat{F}_{s} e^{-\beta \hat{H} / 2} \hat{\mathcal{P}}(t)\right],
$$

where $\hat{F}_{S}(\beta)=e^{-\beta \hat{H} / 2} \hat{F}_{S} e^{-\beta \hat{H} / 2}$ is the Boltzmannized flux operator that is of low rank. ${ }^{15,16}$ It is then straightforward to show that the quantum mechanical activation energy is given by a formula analogous to the classical result in Eq. (7),

$$
E_{a, q m}=\frac{1}{k_{q m}} \lim _{t \rightarrow l o n g} C_{H f s}(t),
$$

where

$$
\begin{aligned}
C_{H f s}(t) & =\frac{1}{2}\left\langle\left[\delta \hat{H}, \hat{F}_{s}(\beta)\right]_{+} \hat{\mathcal{P}}(t)\right\rangle_{q m} \\
& =\frac{1}{2}\left\langle\hat{F}_{s}(\beta)[\delta \hat{H}, \hat{\mathcal{P}}(t)]_{+}\right\rangle_{q m},
\end{aligned}
$$

where $\langle\cdot\rangle_{q m}=\operatorname{Tr}[\cdot] / Q_{r}$ and $\delta \hat{H}=\hat{H}-\langle\mathcal{E}\rangle_{r}$. Here, $[\hat{A}, \hat{B}]_{+}$ $=\hat{A} \hat{B}+\hat{B} \hat{A}$ indicates the anti-commutator and $\langle\mathcal{E}\rangle_{r}$ $=-\partial \ln Q_{r} / \partial \beta$ is the average reactant energy. That is, the activation energy is once again given by a TCF that involves the addition of the fluctuation in the energy at the dividing surface relative to the average reactant energy to the flux-side TCF. The TCF $C_{H f s}(t)$ is symmetrized by our use of the Boltzmannized flux operator in Eq. (17).

The same approach can be used to derive the activation energy based on the rate constant calculated from the quantum mechanical flux-flux TCF,

$$
k_{q m}=\int_{0}^{\infty} C_{f f}(t) d t
$$


where

$$
C_{f f}(t)=\frac{1}{Q_{r}} \operatorname{Tr}\left[\hat{F}_{s}(\beta) \hat{F}_{s}(t)\right] .
$$

Then the activation energy is

$$
\begin{aligned}
E_{a, q m} & =\frac{1}{k_{q m}} \int_{0}^{\infty} \frac{1}{2}\left\langle\left[\delta \hat{H}, \hat{F}_{s}(\beta)\right]_{+} \hat{F}_{s}(t)\right\rangle_{q m} \\
& =\frac{1}{k_{q m}} \int_{0}^{\infty} \frac{1}{2}\left\langle\hat{F}_{s}(\beta)\left[\delta \hat{H}, \hat{F}_{s}(t)\right]_{+}\right\rangle_{q m} .
\end{aligned}
$$

The integrand is a TCF that we will denote as $C_{H f f}(t)$.

Finally, the activation energy can be obtained from a side-side TCF formulation of the quantum mechanical rate constant, ${ }^{4}$

$$
\begin{aligned}
k_{q m} & =\lim _{t \rightarrow \text { long }} \frac{d}{d t} C_{s s}(t) \\
& =\lim _{t \rightarrow \text { long }} \frac{d}{d t}\left\langle e^{-\beta \hat{H}} \theta\left(s^{\ddagger}-\hat{s}\right) \hat{\mathcal{P}}(t)\right\rangle_{q m},
\end{aligned}
$$

where $\theta\left(s^{*}-\hat{s}\right)$ projects onto the reactant space. Symmetrizing the Boltzmann operator as for $C_{f s}(t)$ and $C_{f f}(t)$ above gives the activation energy as

$$
E_{a, q m}=\frac{1}{k_{q m}} \lim _{t \rightarrow l o n g} \frac{d}{d t} C_{H s s}(t),
$$

where

$$
\begin{aligned}
C_{H s s}(t) & =\frac{1}{2}\left\langle\left[\delta \hat{H}, \theta_{\beta}\left(s^{\ddagger}-\hat{s}\right)\right]_{+} \hat{\mathcal{P}}(t)\right\rangle_{q m}, \\
& =\frac{1}{2}\left\langle\theta_{\beta}\left(s^{\ddagger}-\hat{s}\right)[\delta \hat{H}, \hat{\mathcal{P}}(t)]_{+}\right\rangle_{q m},
\end{aligned}
$$

with $\theta_{\beta}\left(s^{\ddagger}-\hat{s}\right)=e^{-\beta \hat{H} / 2} \theta\left(s^{\ddagger}-\hat{s}\right) e^{-\beta \hat{H} / 2}$. We noted above for the classical TCF that the rate constant is equal to the time derivative of $C_{r p}(t)$ for times longer than that required to convert from reactants to products, but we adopted a more global approach to obtaining $k$ from $C_{r p}(t)$. In the quantum mechanical approach described here, the time-derivative is used (as it can be for the classical case as well).

\section{ECKART BARRIER}

We first consider the application of these approaches to direct calculation of the activation energy for a simple test system. The Eckart barrier potential,

$$
V(s)=V_{0} \operatorname{sech}^{2}(s / a),
$$

represents a standard problem in one-dimensional scattering theory; the potential is asymptotically zero at $s \rightarrow \pm \infty$ with a barrier of height $V_{0}$ at $s=0$. The classical rate constant is straightforwardly determined analytically as

$$
k(T)=\sqrt{\frac{k_{b} T}{2 \pi m}} e^{-\beta V_{0}},
$$

which gives the classical activation energy as $E_{a}=V_{0}$ $+k_{b} T / 2$. An exact solution for the quantum mechanical transmission probability is available ${ }^{17}$ from which the rate constant and activation energy can be obtained by the appropriate Boltzmann averaging.

\section{A. Classical dynamics}

For this case we present results for the flux-side approach to calculating the rate constant and activation energy. The flux-side time correlation function, $C_{f s}(t)$, is shown for three different choices of the dividing surface, $s^{\ddagger}$, in Fig. 1. In each calculation, $5 \times 10^{6}$ trajectories are initiated from $s(0)=s^{\ddagger}$ and propagated using the velocity Verlet algorithm at a constant energy that is determined for each trajectory by initial velocities selected from a Boltzmann distribution. These trajectories were then used to evaluate $C_{f s}$ and $C_{H f s}(t)=\left\langle\delta H(0) F_{s}(0) \theta\left[s(t)-s^{\ddagger}\right]\right\rangle$. In the present simulations we took $V_{0}=2.5 k_{b} T, a=1 \AA$, and $m=1 \mathrm{~g} / \mathrm{mol}$; this gives $k=5163 \mathrm{~cm}$ molecule ${ }^{-1} \mathrm{~s}^{-1}$ and $E_{a}=1.79 \mathrm{kcal} / \mathrm{mol}$ at $300 \mathrm{~K}$.

Choosing the dividing surface at $s^{\ddagger}=0$, i.e., at the top of the barrier, yields $C_{f s}(t)=k$ for all $t>0$ because there is no recrossing for this simple one-dimensional potential. This is illustrated in Fig. 1. For $s^{\ddagger}=-2.5$ or $-5 \AA$, however, there is significant recrossing of the (poorly chosen) dividing surface, which can be seen in Fig. 1 by the large initial flux followed by recrossing dynamics that lower the value of $C_{f s}(t)$ at longer times. The recrossing naturally occurs at shorter times for $s^{\ddagger}=-2.5 \AA$ compared to $-5 \AA$ as the
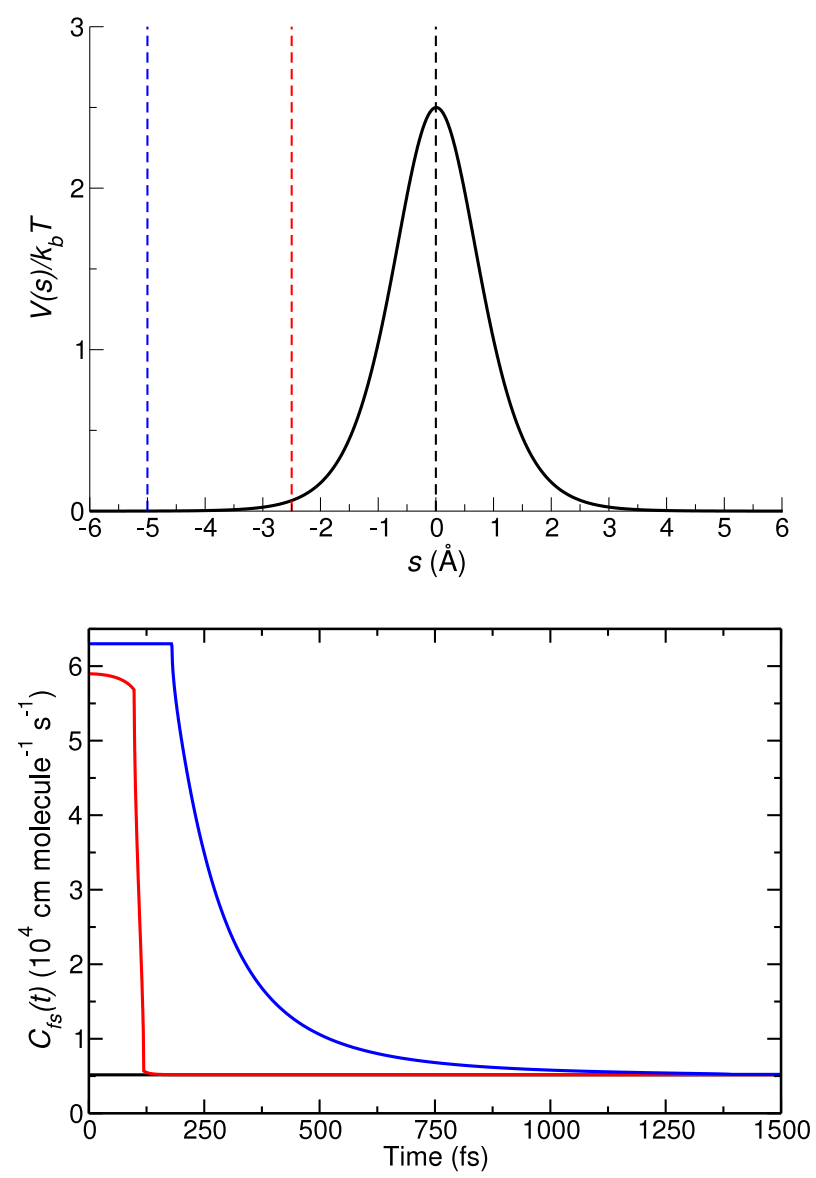

FIG. 1. Top: The Eckart barrier potential is plotted (solid black line) and the three dividing surfaces, $s^{\ddagger}=0,-2.5$, and $-5 \AA$ are shown (vertical dashed black, red, and blue lines, respectively). Bottom: The flux-side TCF, $C_{f s}(t)$, for the one-dimensional Eckart barrier is plotted against time for three choices of the dividing surface: $s^{\ddagger}=0,-2.5$, and $-5 \AA$ (shown as black, red, and blue lines, respectively). 


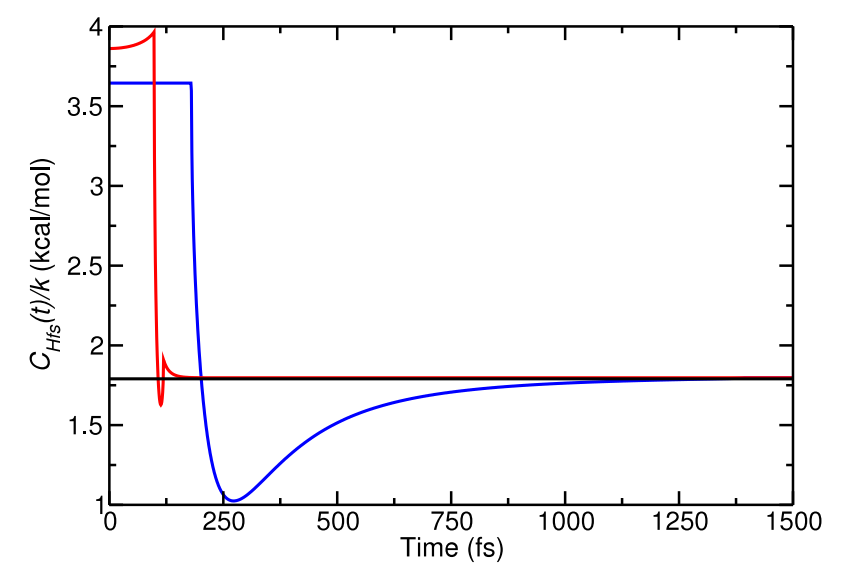

FIG. 2. $C_{H f s}(t) / k$ for the one-dimensional Eckart barrier is plotted against time for three choices of the dividing surface: $s^{\grave{\hbar}}=0,-2.5$, and $-5 \AA$ (shown as black, red, and blue lines, respectively). Normalization by the rate constant in this way means this TCF has a long-time limit equal to the activation energy.

trajectories reach (and are reflected by) the barrier more quickly. The rate constant, given by the long-time limit of $C_{f s}(t)$ is, however, independent of the choice of the dividing surface.

The activation energy can be calculated from the same trajectories from Eq. (7), i.e., it is the long-time limit of $C_{H f s}(t) / k$, which is plotted in Fig. 2 for the three choices of dividing surface location. As with the flux-side TCF itself, $C_{H f s}(t)$ exhibits different dynamics depending on the dividing surface. In the absence of recrossing $\left(s^{\ddagger}=0\right)$ it takes a constant value for all $t>0$. In contrast, for $s^{\ddagger}=-2.5$ and $-5 \AA$, the dividing surface recrossing leads to dynamics on shorter time scales. Specifically, $C_{H f s}(t) / k$ is constant at a value of $\langle H\rangle_{r}=V\left(s^{\ddagger}\right)+k_{b} T / 2$ at short times before the trajectories reach the barrier, are reflected, and recross the dividing surface. The trajectories with the highest kinetic energies recross the dividing surface first, leading to a dip in $C_{H f s}(t) / k$, which then rises steadily to the final value of $E_{a}$ as the trajectories with lower kinetic energy also recross at longer times. It is noteworthy, however, that the activation energy converges on approximately the same time scale as the rate constant. In addition, the same activation energy of $1.79 \mathrm{kcal} / \mathrm{mol}$ is obtained for all choices of $s^{\ddagger}$, in excellent agreement with the analytical result given above.

\section{B. Quantum dynamics}

To illustrate the direct calculation of the quantum mechanical activation energy for the Eckart barrier, we use standard parameters intended to mimic the $\mathrm{H}+\mathrm{H}_{2}$ reaction, $V_{0}=0.425 \mathrm{eV}, a=1$ a.u. $=0.529 \AA$, and $m=1061$ a.u. $=0.5820 \mathrm{~g} / \mathrm{mol}$. The rate constant and activation energy are obtained from the flux-flux TCF formulation, Eqs. (20)-(22). Practically, the low-rank of the Boltzmannized flux operator is used to advantage by finding the two non-zero eigenvalues and eigenvectors,

$$
\hat{F}_{s}(\beta)\left|f_{n}\right\rangle=f_{n}\left|f_{n}\right\rangle
$$

to provide a basis for evaluating the quantum mechanical trace. Then, the flux-flux TCFs $C_{f f}(t)$ and $C_{H f f}(t)$ are obtained as

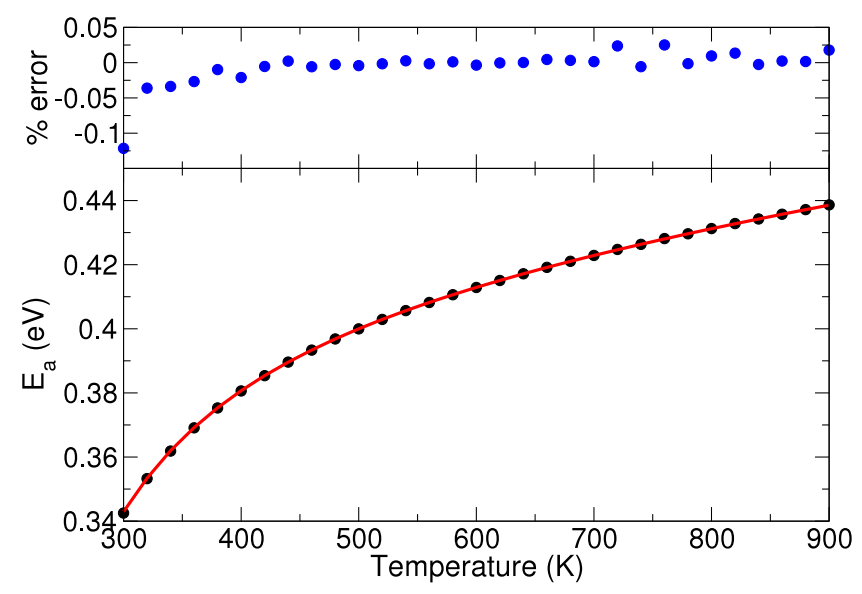

FIG. 3. Bottom: The activation energy for the Eckart barrier calculated using Eq. (22) (black circles) is plotted as a function of temperature and compared with the exact activation energy (red line). Top: The percent-error at each temperature is shown (blue circles).

$$
C_{f f}(t)=\frac{1}{Q_{r}} \sum_{n} f_{n}\left\langle f_{n}\left|\hat{F}_{s}(t)\right| f_{n}\right\rangle
$$

and

$$
C_{H f f}(t)=\frac{1}{2 Q_{r}} \sum_{n} f_{n}\left\langle f_{n}\left|\left[\hat{H}, \hat{F}_{s}(t)\right]_{+}\right| f_{n}\right\rangle
$$

Note that $\left\langle f_{n}\left|\hat{F}_{s}(t)\right| f_{n}\right\rangle=\left\langle f_{n}(t)\left|\hat{F}_{s}\right| f_{n}(t)\right\rangle$ so that the rate constant evaluation involves the time propagation of the eigenvectors $\left|f_{n}\right\rangle$ for which $f_{n} \neq 0$. The only additional work required to obtained $C_{H f f}(t)$, and hence $E_{a, q m}$, is a single additional Hamiltonian multiplication since $\left\langle f_{n}\left|\left[\hat{H}, \hat{F}_{s}(t)\right]_{+}\right| f_{n}\right\rangle=\left\langle f_{n}(t)\left|\hat{H} \hat{F}_{s}+\hat{F}_{s} \hat{H}\right| f_{n}(t)\right\rangle$.

The calculations were completed using a sinc-function discrete variable representation basis ${ }^{18}$ between $s=-10$ and 10 a.u. with a quartic complex absorbing potential starting at $|s|=6$ a.u. with a height of $8 \mathrm{eV}$. The grid spacing was taken to be 15 times the thermal de Broglie wavelength, giving 67 grid points at $300 \mathrm{~K}$ and 117 at $900 \mathrm{~K}$. The Boltzmannized flux eigenvalues and eigenvectors were obtained using a Lanczos algorithm ${ }^{19}$ and the time evolution was carried out with a split-operator propagator with a time step of $0.127 \mathrm{fs}$.

The results for the activation energy, $E_{a, q m}$, as a function of temperature are given in Fig. 3 and compared to the exact results; the error is also shown. The activation energy rises monotonically with temperature beginning from a value below the Eckart potential barrier height $\left(V_{0}=0.425 \mathrm{eV}\right)$. This reduced activation energy can be attributed to quantum mechanical tunneling which occurs due to the low mass used in the calculations. At higher temperatures the activation energy slightly exceeds $V_{0}$. The activation energy calculated directly from Eq. (22) is in excellent agreement with the exact values, differing by less than $0.15 \%$ for all temperatures.

\section{H-BOND EXCHANGES IN WATER}

The final application we consider is the rate constant associated with H-bond exchanges, or "jumps," between different H-bond acceptors in liquid water. ${ }^{20,21}$ Here, the stable-states time correlation function, Eq. (11), is evaluated 
from a single long trajectory that captures a large number of these events. Specifically, TCFs are calculated from a $1 \mathrm{~ns}$ $N V T$ classical MD simulation of 343 water molecules carried out using the LAMMPS software ${ }^{22,23}$ with the SPC/E water force field. ${ }^{24}$ The integration time step was $1 \mathrm{fs}$ and configurations were saved every 2 fs. Coulombic and Lennard-Jones interactions were evaluated within a cut-off radius of $10.5 \AA$. The long-range electrostatic interactions were included using three-dimensional periodic boundary conditions and an Ewald summation with a tolerance of $1 \times 10^{-4}$. The production stage was preceded by 1 ps velocity rescaling and $0.25 \mathrm{~ns} N V T$ equilibration periods. For this system, the reactant state is taken to be a $\mathrm{H}$-bond between a given $\mathrm{OH}$ donor and a particular $\mathrm{O}$ atom $\mathrm{H}$-bond acceptor and the product state is the same $\mathrm{OH}$ $\mathrm{H}$-bonded to a different acceptor; a H-bond is defined geometrically as $R_{\mathrm{OO}} \leq 3.5 \AA, r_{\mathrm{H} \cdots \mathrm{O}} \leq 2.45 \AA$, and $\theta_{\mathrm{OOH}} \leq 20^{\circ}$ and absorbing boundary conditions are used in the product state.

In Fig. 4, we show the reactive time correlation function, $1-C_{r p}(t)$, which decays at longer times with a time constant of $\tau=1 / k=3.1 \mathrm{ps}$, which is consistent with previous determinations of the H-bond jump time in water. $^{21,25}$ Also shown is the $C_{H r p}(t) \equiv\left\langle\delta H(0) n_{r}(0) n_{p}(t)\right\rangle$ TCF that gives the activation energy via Eq. (14). The shape of this $C_{H r p}(t)$ correlation function can be examined by considering the temperature- and time-dependence of $C_{r p}(t)$. It is straightforward to show that if the approximate relationship $1-C_{r p}(t) \simeq e^{-k t}$ holds, then

$$
\frac{d}{d \beta}\left[1-C_{r p}(t)\right]=\left\langle\delta H(0) n_{r}(0) n_{p}(t)\right\rangle \simeq-t \frac{d k}{d \beta} e^{-k t} .
$$

This indicates that $C_{H r p}(t)$ is generally positive (assuming $k$ decreases with increasing $\beta$, i.e., decreasing $T$ ). Moreover, it initially increases with time before decreasing back to zero, with a maximum occurring at $t_{\max }=1 / k$. The value of $C_{H r p}(t)$ at this maximum is easily seen to be

$$
C_{\text {Hrp }}\left(t_{\max }\right) \simeq-\frac{1}{k} e^{-1} \frac{d k}{d \beta}=e^{-1} E_{a} .
$$

Thus, both the rate constant and the activation energy can be directly, if approximately, obtained from the $\left\langle\delta H(0) n_{r}(0) n_{p}(t)\right\rangle$ correlation function using the location

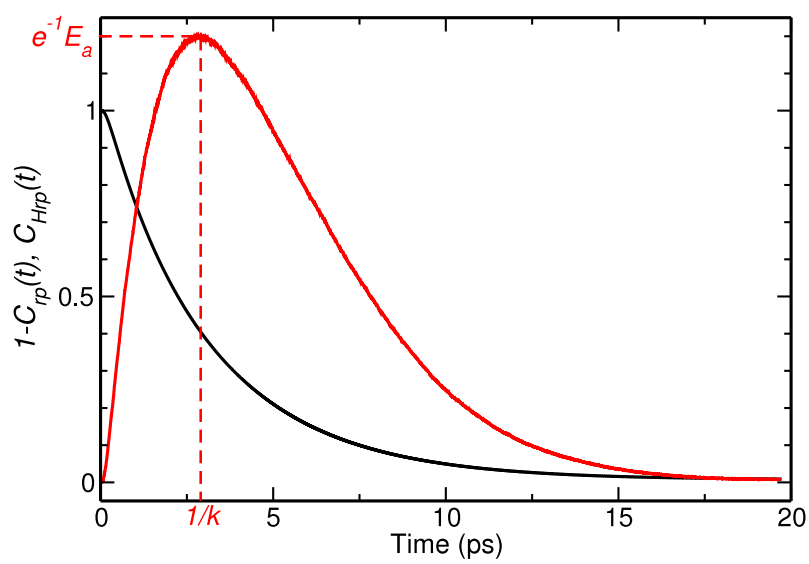

FIG. 4. The stable-states or side-side, reactive TCFs $1-C_{r p}(t)$ (black line) and $C_{H r p}(t)$ (red line) are plotted as a function of time for $\mathrm{H}$-bond exchanges in liquid water. and magnitude of the maximum value. The $C_{H r p}(t)$ in Fig. 4 has a maximum of $1.19 \mathrm{kcal} / \mathrm{mol}$ at $1 / k \simeq 2.9 \mathrm{ps,}$ yielding $E_{a} \simeq 3.2 \mathrm{kcal} / \mathrm{mol}$. This is in good agreement with the rate constant given above and the activation energy value of $E_{a}=2.9 \pm 0.1 \mathrm{kcal} / \mathrm{mol}$ derived from the temperature dependence of the H-bond jump time in water obtained from separate simulations at multiple temperatures.

\section{CONCLUSION}

We have shown that the activation energy for a chemical reaction can be calculated directly within nearly any approach for calculating the rate constant. Because the technique represents a simple extension of standard TCF rate constant computations, it is easily implemented in current classical and quantum mechanical methodologies. More generally, these approaches illustrate how additional information can be directly extracted from reactive trajectories (or quantum dynamics) obtained in simulations.

\section{ACKNOWLEDGMENTS}

Support for this work was provided by the Chemical Sciences, Geosciences, and Biosciences Division, Office of Basic Energy Sciences, Office of Science, U.S. Department of Energy (Grant No. DE-FG02-05ER15708). O.O.M. gratefully acknowledges support from a George E. Walrafen fellowship. The calculations presented were performed at the University of Kansas Advanced Computing Facility. The authors thank Professor James T. Hynes, Professor William H. Miller, and Professor Damien Laage for useful comments on the manuscript.

${ }^{1}$ T. Yamamoto, J. Chem. Phys. 33, 281 (1960).

${ }^{2}$ P. Pechukas and F. J. McLafferty, J. Chem. Phys. 58, 1622 (1973).

${ }^{3}$ W. H. Miller, J. Chem. Phys. 61, 1823 (1974).

${ }^{4}$ W. H. Miller, S. D. Schwartz, and J. W. Tromp, J. Chem. Phys. 79, 4889 (1983).

${ }^{5}$ D. Chandler, J. Chem. Phys. 68, 2959 (1978).

${ }^{6}$ S. H. Northrup and J. T. Hynes, J. Chem. Phys. 73, 2700 (1980).

${ }^{7}$ R. F. Grote and J. T. Hynes, J. Chem. Phys. 73, 2715 (1980).

${ }^{8}$ P. G. Bolhuis, D. Chandler, C. Dellago, and P. L. Geissler, Annu. Rev. Phys. Chem. 53, 291 (2002).

${ }^{9}$ C. Dellago and P. G. Bolhuis, Mol. Simul. 30, 795 (2004).

${ }^{10}$ C. S. Lo, R. Radhakrishnan, and B. L. Trout, Catal. Today 105, 93 (2005).

${ }^{11}$ E. E. Borrero and C. Dellago, J. Chem. Phys. 133, 134112 (2010).

${ }^{12}$ C. Drechsel-Grau and M. Sprik, J. Chem. Phys. 136, 034506 (2012).

${ }^{13}$ R. C. Tolman, J. Am. Chem. Soc. 42, 2506 (1920).

${ }^{14}$ This form also assumes the use of absorbing boundary conditions wherein $n_{p}(t)=1$ for all times $t$ after a trajectory reaches the product region, i.e., after $s(t)$ passes $s_{p}$. In this way, longer-time dynamics associated with reformation of the reactants from the products through the reverse reaction are disregarded.

${ }^{15}$ T. J. Park and J. C. Light, J. Chem. Phys. 88, 4897 (1988).

${ }^{16}$ W. H. Thompson and W. H. Miller, J. Chem. Phys. 106, 142 (1997).

${ }^{17}$ H. S. Johnston, Gas Phase Reaction Rate Theory (Ronald Press, New York, 1966).

${ }^{18}$ D. T. Colbert and W. H. Miller, J. Chem. Phys. 93, 1982 (1992).

${ }^{19}$ C. Lanczos, J. Res. Natl. Bur. Stand. 45, 255 (1950).

${ }^{20}$ D. Laage and J. T. Hynes, Science 311, 832 (2006).

${ }^{21}$ D. Laage and J. T. Hynes, J. Phys. Chem. B 112, 14230 (2008)

${ }^{22}$ S. J. Plimpton, J. Comput. Phys. 117, 1 (1995).

${ }^{23}$ The LAMMPS molecular dynamics package, http://lammps.sandia.gov.

${ }^{24}$ H. J. C. Berendsen, J. R. Grigera, and T. P. Straatsma, J. Phys. Chem. 91, 6269 (1987).

${ }^{25}$ A. A. Vartia, K. R. Mitchell-Koch, G. Stirnemann, D. Laage, and W. H. Thompson, J. Phys. Chem. B 115, 12173 (2011). 